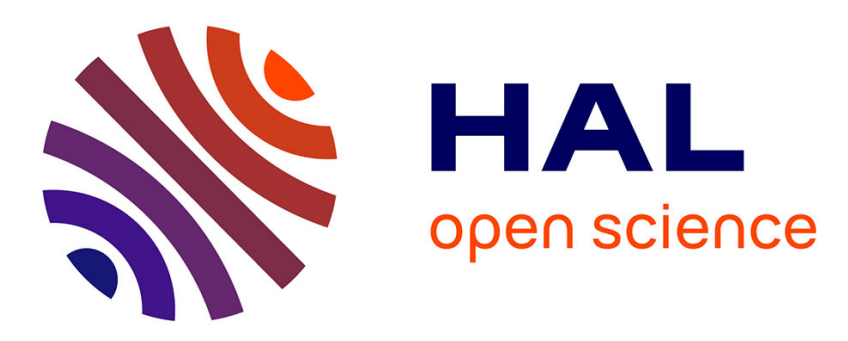

\title{
Répartition des métaux lourds dans les différentes fractions des sédiments marins: influence de la protection cathodique
}

Lydia Leleyter, Christelle Rousseau, Otavio Gil, Fabienne Baraud

\section{- To cite this version:}

Lydia Leleyter, Christelle Rousseau, Otavio Gil, Fabienne Baraud. Répartition des métaux lourds dans les différentes fractions des sédiments marins: influence de la protection cathodique. Comptes Rendus Géoscience, 2007, 339 (1), pp.31-39. 10.1016/j.crte.2006.10.004 hal-02587751

\section{HAL Id: hal-02587751}

https://hal-normandie-univ.archives-ouvertes.fr/hal-02587751

Submitted on 27 May 2020

HAL is a multi-disciplinary open access archive for the deposit and dissemination of scientific research documents, whether they are published or not. The documents may come from teaching and research institutions in France or abroad, or from public or private research centers.
L'archive ouverte pluridisciplinaire HAL, est destinée au dépôt et à la diffusion de documents scientifiques de niveau recherche, publiés ou non, émanant des établissements d'enseignement et de recherche français ou étrangers, des laboratoires publics ou privés. 


\section{Page de titre}

\section{Rubrique : Comptes Rendus Géoscience}

Répartition des métaux lourds dans les différentes fractions des sédiments marins : influence de la protection cathodique

Influence of cathodic protection on heavy metals partitioning in marine sediments

\section{Lydia Leleyter, Christelle Rousseau, Otavio Gil et Fabienne Baraud}

Equipe de Recherche en Physico-chimie et Biotechnologies / CORRODYS - EA 3914

Sciences 2, Campus 2, Bd du Maréchal Juin, 14032 Caen, France

Corresponding author : Tel : 02.31.56.72.18-Fax : 02.31.56.73.03-Mail : lydia.reinert@chimie.unicaen.fr

\section{Résumés courts}

Abstract : A large scale experiment, with natural seawater and sediments, was conducted during one month, to evaluate the possible interaction of cathodic protection $(\mathrm{E}=-1 \mathrm{~V} / \mathrm{Ag}-\mathrm{AgCl})$ on the distribution patterns of trace elements present in the sediments. No influence on metals lability and distribution within marine sediments was observed. Nevertheless, the sacrificial anode dissolution induces a significant zinc concentration increase in the superficial sediments. This accumulated zinc is mainly scavenged in labile fractions (acido-soluble and reducible) of the sediments.

Keywords : sediments / fractionation / sacrificial anode / heavy metals / zinc 
Résumé : Une expérience en conditions semi-naturelle a mis en évidence l'absence d'influence notable de la proximité d'un acier placé, pendant un mois, sous protection cathodique (E=-1V/Ag$\mathrm{AgCl})$ sur la labilité et sur la spéciation des éléments traces présents dans des sédiments marins. Par ailleurs, la dissolution des anodes sacrificielles en zinc a conduit à une augmentation de la concentration en zinc dans les sédiments directement en contact avec la phase aqueuse. Plus précisément, le zinc libéré est stocké majoritairement dans deux fractions labiles des sédiments : les fractions acido-solubles et réductibles.

Mots clefs : sédiments / spéciation minéralogique / anode sacrificielle / métaux lourds / zinc 


\section{$\underline{\text { Abridged version }}$}

\section{Introduction}

Steel structures exposed to sea water are often protected against corrosion by cathodic protection. The process often used involves realising an electrical connection between steel and a block of a least noble metal (as zinc) which behaves as an anode, in order that the steel behaves as a cathode. The resulting anode dissolution contributes to input of zinc to coastal waters which may cause ecological damage, as zinc is recognized to be toxic to a variety of biota, at early life stage or inducing chronic toxicity (Matthiessen et al, 1999; Jelmert et Van Leeuwen, 2000). As sediments present various scavenging surface sites, much of the released zinc, from anodes, is likely to be transferred to sediment phase (Bird et al., 1996). Therefore, adsorbed elements onto solid particles are potentially available as they may be dissolved due to changes in the physico-chemical properties of the environment (Abollino et al., 2002, Leleyter and Probst, 1999; Bourg, 1995; Forstner, 1993). Many chemical extraction (leaches) procedures have been proposed in the literature to estimate the mobility of heavy metals in sediments in order to determine their bioavailability. Thus, sequential extraction procedures (several steps) are used to differentiate mobile and residual fractions, and to characterize the different labile fractions (Leleyter et Probst, 1999, Ure et al.,1995 ; Schuman, 1985 ; Tessier et al., 1979): exchangeable fraction (weakly bound elements in equilibrium with the dissolved phase), acidosoluble fraction (very sensitive to $\mathrm{pH}$ variations), reducible or oxidable fractions (very sensitive to redox conditions and microbiological activity). Sequential extractions are then useful for solid partitioning of particulate elements, to study the origin, the fate, the biological and physicochemical availability and transport of sorbed elements. The aims of this work are to:

- study the fate of zinc solubilized from sacrificial anodes.

- evaluate the possible influence of imposed potential on heavy metals fractionation in marine sediments in contact with steel under cathodic protection.

\section{Materials and methods}

\subsection{Cathodic protection with zinc sacrificial anode}


During 1 month, at the marine station of Luc-sur-Mer (Normandy, France), an experiment was conducted in a large-scale tank (about 860L), supplied with decanted natural sea water (stable $\mathrm{pH}$, equal to 8.1). A $70 \mathrm{~L}$ container, filled with natural marine sediments (particles diameter $<60 \mu \mathrm{m}$ ) is immersed in the tank (Figure 1). Some steel samples are buried in the sediments and others are immersed in the water. All the samples are corrosion-protected by sacrificial zinc anodes. The resulting imposed potential is $-1 \mathrm{~V} / \mathrm{Ag}-\mathrm{AgCl}$. Water temperature is maintained at $14^{\circ} \mathrm{C}$. Sediments and water samples are collected at the beginning and at the end of the experiment.

\subsection{Sediments characterisation}

Total concentrations of elements in sediments were determined after microwave assisted acid digestion $\left(\mathrm{HNO}_{3}\right.$ and $\left.\mathrm{HCl}\right)$, by Inductively Coupled Plasma Atomic Emission Spectrometry (ICPAES) (Varian Vista-MPX). Sediments were also leached by an optimized sequential chemical extraction procedure (Leleyter and Probst, 1999). This procedure selectively and efficiently dissolves all the chemical constituents of the sediments, in the following order: S1: water soluble, S2: exchangeable, S3: acido-soluble, S4: reducible, S5: oxidable fractions and S6: sum of all these labile fractions.

\section{Results and discussion}

\subsection{Sea water chemical composition}

Despite sacrificial anodes dissolution, no significant increase of zinc concentration was detected (table 1), which might be due to an important dilution phenomena and/or zinc accumulation in sediments (Bird et al. 1996 ; table 3).

\subsection{Marine sediments composition}

4 collected sediments were analysed :

- LOH : superficial sediment (beginning of experiment)

- LOM : sediment in contact with steel (beginning of experiment)

- L1H : superficial sediment (end of experiment) 
- L1M : sediment in contact with steel (end of experiment)

Major element concentrations in collected sediments present slight $\mathrm{Ca}$ and $\mathrm{Fe}$ enrichments, if one compares with the baseline values proposed by Stevenson (2001) for marine sediments (table 2) and, as a compensation, are depleted in aluminium, magnesium and potassium. This calcium enrichment is due to marine shells erosion (which contain calcium carbonate) collected with our sediments. The low aluminium content reflects relative low clay minerals content of these sediments. No spatial nor temporal evolution in major chemical composition of the sediments was noticed, except for calcium, as its concentration was lower at the end of the experiment, in the superficial sediment $(\mathrm{L} 1 \mathrm{H})$.

Trace elements concentrations present in the initial sediments are reported in table 3 and compared to the baseline values proposed by Stevenson (2001) for non-polluted sediments and to the criteria proposed by the 14/06/00 french decree. It appears that these sediments can be considered as moderately polluted, mainly in $\mathrm{Pb}, \mathrm{Zn}$ and $\mathrm{Cu}$.

After one month experiment, no spatial nor temporal evolution of the metals concentrations in sediments was observed, for all the studied elements excepted zinc (table 3). Indeed, a much higher total $\mathrm{Zn}$ concentration is detected in the final superficial sediment $(\mathrm{L} 1 \mathrm{H})$. This zinc enrichment results from sacrificial anode dissolution. This could be correlated to the observed Ca decrease, supposing some cationic exchange processes between these two elements, between superficial sediments and seawater. No influence of sacrificial anodes dissolution was detected on deeper sediments.

\subsection{Heavy metal partitioning}

In agreement with the literature (Gaiero et al. 2003), the most important heavy metals scavenged fractions are the acido-soluble (S3), reducible (S4) and oxidable (S5) fractions (table 4).

This study points out that the proximity of steel under cathodic protection had no influence on the elements lability (except may be calcium and copper). The percentages of non-residual element, for all the studied elements, show no spatial nor temporal variation. This result is surprising when focusing on zinc, really more concentrated in the $\mathrm{L} 1 \mathrm{H}$ sediment. It can be concluded that a part of the released zinc (from sacrificial anode) is irreversibly absorbed into the residual fraction of the sediment. 
Moreover, except for zinc, the elements fractionations present no variation between sediment in contact with steel under cathodic protection (L1M) and other sediments. The potential did not influence the elements partitioning between oxide and reducible fraction in sediments (table 4).

Focusing on zinc partitioning, it appears that the solubilized zinc is mainly scavenged into the acidosoluble (S3) and the reducible (S4) fractions (table 4, figure 2). This kind of distribution is not unusual for $\mathrm{Zn}$ and has been reported in various studies (Morillo et al, 2004 ; Usero et al., 1998 ; Adamo et al., 2005 ; Lopez-Sanchez et al., 1996). This was quite surprising for the Zn-contaminated sample, as one could expect that the released zinc would have been firstly accumulated in the water soluble fraction. But no zinc was leached in the S1 fraction.

As zinc can exert chronic toxicity to certain crustaceans (Matthiessen et al, 1999), it might be keept in mind that the fate of solubilized zinc (from sacrificial anode) is to scavenge into labile (acido-soluble and reducible) fractions (so bioavailable fractions) of the sediments.

\section{Conclusion}

This study points out that the proximity of steel under cathodic protection $(\mathrm{E}=-1 \mathrm{~V} / \mathrm{Ag}-\mathrm{AgCl})$, during one month, has no influence on the elements lability nor on the elements fractionation, in a slightly polluted sediment.

Moreover, an important increase of zinc concentration in superficial sediments was noticed due to the sacrificial anode dissolution. A part of this dissolved zinc is, irreversibly, absorbed into the residual fraction of the sediment, but the main part of this solubilized zinc is scavenged into labile (acidosoluble and reducible) fractions of the sediments.

\section{Version francaise}

\section{Introduction}

Les ouvrages métalliques immergés en milieu marin, notamment les infrastructures portuaires, sont souvent protégés contre la corrosion par protection cathodique. Le principe est d'amener la surface de la structure métallique à un potentiel assez négatif pour rendre l'acier suffisamment cathodique et donc 
non corrodable, en lui permettant de rester dans son domaine d'immunité. Deux méthodes de mise en œuvre existent :

- protection par anode sacrificielle : l'anode est constituée d'un métal (souvent essentiellement en zinc) à un potentiel moins élevé que celui de l'acier. Sa fixation sur l'acier (cathode), à protéger, assure un bon contact électrique et ainsi un courant d'électrons entre l'anode et la cathode.

- courant imposé par générateur externe: les électrons sont produits par un courant électrique provenant d'une source externe (générateur) qui impose une charge négative à l'acier, ce qui crée une réaction cathodique et ainsi protège l'acier.

D'un point de vue environnemental, la protection cathodique par anode sacrificielle en zinc est extrêmement polluante ; la solubilisation des anodes contribue grandement à l'augmentation de la concentration en zinc notamment en zone portuaire (Matthiessen et al, 1999 ; Jelmert et Van Leeuwen, 2000). Or le zinc peut entraîner des maladies chroniques chez certains mollusques et crustacés (Matthiessen et al, 1999). Une fois libéré en phase aqueuse, le zinc s'accumule dans les sédiments portuaires, les contaminant à leur tour. Ainsi, des concentrations multipliées par 2,5 par rapport au bruit de fond local, ont été reportées pour des sédiments portuaires (Bird et al., 1996). Cependant, ces phénomènes de "sorption" (absorption ou adsorption) pouvant être réversibles, tous les éléments métalliques "sorbés" sur un sédiment sont potentiellement remobilisables, lors du changement d'un paramètre physico-chimique tel que la salinité, le $\mathrm{pH}$, le potentiel d'oxydo-réduction et/ou la concentration en ligands (Abollino et al., 2002, Leleyter and Probst, 1999; Bourg, 1995; Forstner, 1993). Or, le risque écologique est plus particulièrement lié à la forme chimique de l'élément, à sa mobilité et à sa biodisponibilité (définie, selon Baize (1997), comme l'aptitude d'un élément à passer d'un compartiment quelconque du sol dans un être vivant; cette définition caractérise une aptitude, plus que le déroulement effectif du phénomène). Ainsi, l'évaluation des risques liés à une pollution métallique d'un sédiment ou d'un sol ne dépend pas uniquement de la quantité totale de métaux détectée dans le milieu. De même, une pollution métallique de la phase aqueuse doit être évaluée non seulement de la quantité totale de métaux présents, mais aussi en fonction de leur disponibilité ; celleci est notamment accessible par la technique du gradient de diffusion en couches minces (DGT), qui distingue le métal dissout labile (qui comprend l'ion métallique libre plus les complexes organiques et 
inorganiques pouvant se dissocier pendant le laps de temps nécessaire pour la diffusion à travers l'hydrogel) du métal dissout inerte (Buzier et al. 2006).

Pour les sols et les sédiments, de nombreuses techniques (extractions chimiques simples ou séquentielles, ou études par spectroscopie EXAFS : Extended X-Ray Absorption Fine Structure, Kirpichtchikova et al. 2006) sont proposées dans la littérature afin d'estimer l'importance de la fraction mobilisable (ou "labile") des métaux présents dans des sols ou des sédiments, voire d'essayer d'appréhender leur biodisponibilité. Les extractions séquentielles, multi-étapes (Leleyter et Probst, 1999, Ure et al.,1995 ; Schuman, 1985 ; Tessier et al., 1979), tout comme la spectroscopie EXAFS, permettent de différencier non seulement la fraction globalement labile, de la fraction résiduelle, mais aussi de préciser la répartition des éléments dans différentes fractions des sols ou sédiments susceptibles d'être solubilisées dans le milieu naturel lors du changement d'un des paramètres physico-chimiques de la phase aqueuse. Elles sont ainsi des outils extrêmement performants pour prédire l'origine, le devenir, la mobilité voire la biodisponibilité des éléments (Leleyter and Baraud, 2005).

Ainsi, les éléments liés à la fraction réellement échangeable, c'est à dire les éléments situés essentiellement dans l'espace interfolliaire des minéraux argileux, sont très labiles. Ils sont susceptibles d'être solubilisés lors d'une diminution du pH ou d'une augmentation de la salinité. Les éléments solubilisés avec la fraction acido-soluble seront, comme leur nom l'indique, très sensibles à une diminution du pH. Tandis que les éléments piégés par les fractions oxydables (essentiellement composées de matière organique) et réductibles (essentiellement les oxydes de fer et de manganèse) seront eux plus sensibles aux variations du potentiel d'oxydo-réduction.

Les objectifs de cette étude sont de :

- suivre le devenir du zinc issu de la dissolution des anodes sacrificielles.

- évaluer l'éventuelle influence d'un potentiel imposé sur la répartition des éléments (essentiellement métaux lourds) dans les différentes fractions minéralogiques des sédiments au contact de pièces métalliques placées sous protection cathodique.

Une expérience à grande échelle, en condition semi naturelle, a été lancée dans un bassin de 860 litres de la station marine de Luc-sur-Mer (Normandie, France). Ce bassin est alimenté en eau de mer 
naturelle décantée et contenant un bac de sédiments naturels. Des échantillons d'acier y sont soit immergés dans l'eau, soit enterrés dans les sédiments. La protection cathodique est assurée à l'aide d'anodes sacrificielles en zinc. Les prélèvements de sédiments et d'eau de mer sont réalisés en début d'expérience et après 1 mois d'essai.

\section{Matériels et méthodes}

\subsection{Protection cathodique par anode sacrificielle}

Les essais sont réalisés dans un bassin de 380 x 47 x 48cm (environ 860 L), alimenté en eau de mer naturelle prélevée à $300 \mathrm{~m}$ du rivage (pH stable, égal à 8,1), décantée, en circuit fermé mais renouvelable, à hauteur de 5\%. Un bac contenant environ 70L de sédiments marins naturels (particules de diamètre $<60 \mu \mathrm{m}$ ), provenant de la décantation de l'eau de mer utilisée, est placé dans le bassin (figure 1). Les échantillons d'acier (5 x $5 \mathrm{~cm}$ ) sont regroupés en 10 modules de 14 échantillons dont la moitié est enterrée dans le bac de sédiments. Les autres modules sont immergés dans le même bassin. La température de l'eau est maintenue à $14^{\circ} \mathrm{C}$ à l'aide d'un thermostat. La protection cathodique est assurée par le couplage de chaque module à une anode sacrificielle en zinc. Le potentiel appliqué est d'environ -1V/Ag-AgCl.

\subsection{Analyse des sédiments}

\subsubsection{Concentrations totales}

Les sédiments prélevés sont séchés à l'étuve à $37^{\circ} \mathrm{C}$ pendant 4 jours, puis broyés dans un mortier en agate. Les teneurs totales sont déterminées par Spectromètre d'Emission Atomique Couplé à un Plasma Induit (ICP-AES) (Varian Vista-MPX), après une minéralisation acide à l'eau régale $(10 \mathrm{~mL}$ pour 0,2g de sédiment sec) assistée par micro-ondes (Berghof, Speedwave MSW2).

\subsubsection{Extraction séquentielle}

La répartition des métaux lourds dans les sédiments est déterminée grâce à un protocole d'extraction séquentielle selon Leleyter et Probst (1999). Ce protocole permet de dissoudre, de manière sélective et 
efficace, chacune des fractions du sédiment susceptible d'être affectée par un changement de composition physico-chimique de la phase aqueuse, à savoir : la fraction soluble à l'eau (S1), la fraction réellement échangeable (S2 : composée essentiellement des éléments situés dans l'espace interfolliaire des minéraux argileux); la fraction acido-soluble (S3: essentiellement formée de carbonates et de phosphates (Leleyter et Baraud, 2006)) ; la fraction réductible (S4) (oxydes de manganèse : S4a; oxydes de fer amorphes : S4b et oxydes de fer cristallins : $\mathrm{S} 4 \mathrm{c}$ ) et la fraction oxydable (S5: essentiellement formée de matière organique). La somme de toutes ces fractions lessivables représente la fraction labile du sédiment, qui est notée S6. La concentration de la fraction résiduelle est obtenue par différence entre la concentration totale initiale et celle obtenue par la somme des fractions labiles (S6).

\section{Résultats et discussion}

\subsection{Phase aqueuse}

La dissolution des anodes sacrificielles laisse présager d'une augmentation de la teneur en zinc dans l'eau. Or, les concentrations, déterminées par ICP-AES, ne montrent pas de variation significative après un mois d'essai, ni pour Zn, ni pour l'ensemble des éléments analysés (tableau 1). Ce résultat peut être attribué à une dilution importante, liée au renouvellement continuel de l'eau de mer et/ou à l'accumulation du zinc libéré par les anodes dans les sédiments (Bird et al. 1996 ; tableau 3).

\subsection{Composition des sédiments}

Afin de suivre l'interaction entre la protection cathodique et les sédiments, 4 prélèvements de sédiments ont été analysés :

- $\quad$ LOH : prélèvement superficiel en début d'essai

- $\quad$ LOM : prélèvement entre 2 plaques métalliques en début d'essai

- $\quad$ L1H : prélèvement superficiel après 1 mois d'expérience

- $\quad$ L1M : prélèvement entre 2 plaques métalliques après 1 mois d'expérience 
Les tableaux 2 et 3 donnent la composition des sédiments secs collectés. Ces sédiments sont initialement enrichis en calcium et en fer, si on se réfère aux valeurs de référence proposées par Stevenson (2001), et, en compensation appauvris, notamment en aluminium. Cet enrichissement en calcium provient sans doute de l'érosion des coquillages marins (dans lesquels le calcium est présent sous forme de carbonate de calcium) présents dans les sédiments collectés (hypothèse confirmée, après une analyse par calcimétrie Bernard, selon laquelle les sédiments étudiés contiennent initialement 33\% de carbonates de calcium). La faible concentration en aluminium indique une faible teneur de nos sédiments en minéraux argileux. La seule variation significative après 1 mois d'essai est une diminution de la teneur en calcium dans les sédiments collectés en surface (L1H).

Les teneurs en cadmium, cobalt, chrome, cuivre, nickel, plomb et zinc des sédiments prélevés au début de l'essai, sont toutes inférieures à celles du niveau 1 de l'arrêté du 14 juin 2000 relatif aux niveaux de référence à prendre en compte lors de l'analyse de sédiments marins ou estuariens présents en milieu naturel ou portuaire. Cependant on note que les teneurs en cuivre, plomb et zinc sont supérieures aux moyennes rapportées, dans les régions Atlantiques et de la Mer du Nord, par Stevenson (2001) pour des sédiments non contaminés. On peut donc considérer que les sédiments utilisés lors de cette expérience sont initialement peu contaminés.

Comme pour les éléments majeurs, après un mois d'expérience, aucune influence notable, spatiale ou temporelle, n'est relevée pour la majorité des éléments étudiés. Seule la teneur en zinc augmente de façon importante dans les sédiments collectés en surface (L1H). Cette augmentation provient, sans nul doute, de la dissolution des anodes sacrificielles. Il semble donc que le zinc libéré par les anodes sacrificielles s'accumule dans les sédiments de surface sans avoir le temps, lors de cette étude, de contaminer les sédiments plus en profondeur.

Cette augmentation peut-être mise en parallèle avec la diminution en calcium dans cette zone (L1H). On peut supposer que des phénomènes d'échanges cationiques $\mathrm{Ca} / \mathrm{Zn}$ se sont produits entre les sédiments de surface et la phase aqueuse.

\subsection{Distribution des métaux lourds}


Conformément à la littérature (Gaiero et al. 2003), les fractions acido-solubles (S3), réductibles (S4) et oxydables (S5) restent les fractions préférentielles de stockage des métaux lourds dans les sédiments (tableau 4).

Cette étude met en évidence l'absence d'influence notable de la proximité de l'acier sous protection cathodique sur la labilité des éléments présents dans les sédiments à l'exception du $\mathrm{Ca}$ et $\mathrm{Cu}$ qui semblent légèrement sensibles. En effet, pour tous les éléments étudiés, le pourcentage d'élément labile ne montre ni évolution temporelle, ni évolution spatiale (écart type inférieur à 10\%). Ce résultat reste vrai pour le zinc sur l'échantillon $\mathrm{L} 1 \mathrm{H}$, qui présente des teneurs totales 5 fois plus importantes. Ce résultat est très surprenant: on aurait pu s'attendre à ce que tout le zinc accumulé soit labile, ce qui ce serait traduit par une augmentation de sa labilité totale dans L1H. Il semble donc qu'une partie du zinc libéré par les anodes s'absorbe, de manière irréversible, dans les sédiments.

De plus, la répartition des éléments (excepté le zinc) dans les différentes fractions minéralogiques des sédiments (notamment les fractions réductibles et oxydables) ne semble pas non plus être influencée par la proximité d'acier sous protection cathodique. En effet, il n'apparaît pas de différence significative entre les pourcentages obtenus, dans les deux fractions minéralogiques par définition sensibles au potentiel (S4 et S5), entre le sédiment L1M et les autres (tableau 4).

La distribution du zinc est plus particulièrement intéressante. De façon générale, pour l'ensemble des échantillons, le zinc se trouve de façon prédominante dans les fractions acido-soluble (S3) et réductible (S4). Ce type de distribution n'est pas inhabituel et est rapporté par diverses études, pour des sédiments marins contaminés ou non (Morillo et al, 2004 ; Usero et al., 1998 ; Adamo et al., 2005 ; Lopez-Sanchez et al., 1996)

Il s'avère néanmoins que le zinc apporté par les anodes sacrificielles a pour site de stockage prépondérant la fraction acido-soluble (S3), tandis que les échantillons qui ne sont pas touchés par cet apport exhibent un site de stockage préférentiel dans la fraction réductible (S4) (tableau 4, figure 2). Or, on aurait pu imaginer qu'une partie du zinc libéré se dépose juste sur les sédiments, sans phénomènes d'adsorption ou d'absorption, et soit donc lessivable à l'eau. Or il n'en est rien car la fraction S1 ne libère pas de zinc. 
Par ailleurs, il est important de souligner que si la répartition du zinc dans les différentes fractions du sédiment contaminé par la dissolution des anodes varie peu en terme de pourcentage mobilisé, les quantités absolues (en $\mu \mathrm{g} / \mathrm{g}$ ) de zinc labile sont beaucoup plus importantes ( 5 à 10 fois plus élevées suivant les fractions considérées, cf. figure 2). Ceci peut laisser présager d'un relargage important de zinc (potentiellement biodisponible) dans la phase aqueuse et de pic de concentration pouvant être localement toxique. Sachant que le zinc peut induire une toxicité chronique chez certains mollusques et crustacés (Mathiessen et al.1999), ce résultat est à garder en mémoire.

\section{Conclusion}

Ce travail a mis en évidence l'absence d'influence notable de la proximité d'un acier placé, pendant un mois, sous protection cathodique $(\mathrm{E}=-1 \mathrm{~V} / \mathrm{Ag}-\mathrm{AgCl})$ sur la labilité et sur la répartition des éléments présents dans des sédiments marins peu pollués.

Parallèlement, les résultats montrent que la dissolution des anodes sacrificielles en zinc conduit à une augmentation de la concentration en zinc dans les sédiments marins directement en contact avec la phase aqueuse. Une fraction de ce zinc semble s'accumuler dans la fraction résiduelle des sédiments, mais une majorité s'associe avec la fraction labile (donc potentiellement biodisponible). Plus précisément, le zinc libéré par les anodes sacrificielles est stocké majoritairement dans deux fractions labiles des sédiments : les fractions acido-solubles et réductibles. Il semble préférable, d'un point de vue environnemental, de faire une protection cathodique par courant imposé plutôt que par anode sacrificielle (méthode qui implique nécessairement un relargage, dans le milieu naturel, de quantités importantes d'espèces métalliques, le plus souvent l'aluminium ou comme ici, le zinc. Cette pollution est d'autant plus préoccupante (notamment en zone portuaire ou à proximité des côtes) qu'elle concerne la fraction mobile des sédiments. Des expériences complémentaires, à plus long terme et sur des sédiments composés d'argiles pures, actuellement développées au laboratoire, permettront d'enrichir les connaissances actuelles sur le devenir du zinc, libérés par les anodes sacrificielles. 


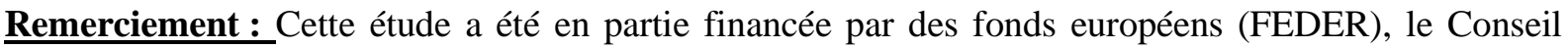
Régional de Basse Normandie et le Conseil Général de la Manche.

\section{$\underline{\text { Références }}$}

Abollino O., Aceto M., Malandrino M., Mentasti E., Sarzanini C. and Barberis R., Distribution and mobility of metals in contaminated sites, Chemometric investigation of polluant profiles, Environmental Pollution 119 (2002) 177-193.

Adamo P., Arienzo M., Imperato M., Naimo D., Nardi G. and Stanzione D., Distribution and partition of heavy metals in surface and sub-surface sediments of Naples city port, Chemosphere 61 (2005) 800-809

Arrêté du 14 juin 2000 relatif aux niveaux de référence à prendre en compte lors d'une analyse de sédiments marins ou estuariens présents en milieu naturel ou portuaire, Journal Officiel du 10 août 2000.

Baize D., Teneurs totales en éléments traces métalliques dans les sols (France), Un point sur. ISSN : 1250_5218; ISBN : 2738007473.INRA, Paris, 1997, 409 p.

Bird P., Comber S.D.W., Gardner M.J. and Ravenscroft J.E., Zinc inputs to coastal waters from sacrificial anodes. The Science of the Total Environment, 181(1996) 257-264.

Bourg A.C.M., Speciation of heavy metals in soils and groundwater and implications for their natural and provoked mobility. In Heavy metals : problems and solutions, Salomons W., Forstner U. and Mader P. eds., Springer Berlin, Federal Republic of Germany, 1995, pp 19-32.

Buzier R., Tusseau-Vuillemin M.H., Mouchel J.M. , Evaluation of DGT as a metal speciation tool in wastewater, Science of the Total Environment 358 (2006) 277-285

Forstner U., Metal speciation : general concepts and applications, International Journal of Environmental Analytical Chemistry, 51 (1993) 5-23. 
Gaiero D.M., J-L. Probst, P.J. Depetris, S. Bidart and L. Leleyter, Iron and other transition metals in Patagonian riverborne and windborne materials: their geochemical control and transport to the SW Atlantic ocean. Geochimica et Cosmochimica Acta. 67 (2003) 3603-3623.

Jelmert A. and van Leeuwen J.H., Harming local species or preventing the transfer of exotics? Possible negative and positive effects of using zinc anodes for corrosion protection of ballast water tanks, Water Research 34 (6) (2000) 1937-1940.

Kirpichtchikova T.A., Manceau A., Spadini L., Panfili F., Marcus M.A., Jacquet T., Speciation and solubility of heavy metals in contaminated soil using X-ray microfluorescence, EXAFS spectroscopy, chemical extraction and thermodynamic modelling, Geochemica et Cosmochimica Acta 70 (2006) 2163-2190

Leleyter L. et Baraud F., 2006 Selectivity and efficiency of the acido-soluble extraction in sequential extraction procedure, International Journal of Soil Sciences (accepté).

Leleyter L. and Baraud F., Evaluation de mobilité des métaux dans les sédiments fluviaux du bassin de la Vire (Normandie, France) par extractions simples ou séquentielles, Evaluation of metals mobility in sediments by single or sequential extractions. C. R. Geosciences. 337 (2005) 571-579.

Leleyter L. and J-L. Probst, A new sequential extraction procedure for the speciation of particulate trace elements in river sediments, Int. J. Environ. Anal. Chem., 73 (1999) 109-128.

Lopez-Sanchez J.F., Rubio R., Samitier C.and Rauret G., Trace metal partitioning in marine sediments and sludges deposited off the coast of Barcelona (Spain), Water Research 30(1) (1996) 153-159

Matthiessen P., Reed J. and Johnson M., Sources and potential effects of copper and zinc concentrations in the estuarine waters of Essex and Suffolk, United Kingdom. Marine Pollution Bulletin 38 (1999) 908-920.

Morillo J., Usero J. and Gracia I., Heavy metal distribution in marine sediments from the southwest coast of Spain, Chemosphere 55 (2004) 431-442.

Schuman L.M., Fractionation method for soil microelements, Soil Science, 140 (1985) 11-22.

Stevenson A.G., Metal concentrations in marine sediments around Scotland: a baseline for environmental studies, Continental Shelf Research, 21 (2001) 879-897 
Tessier A., Campbell P.G.C. and Bisson M, Sequential extraction procedure for speciation of particulate trace metals, Anlytical Chemistry, 51 (1979) 844-851.

Ure A.M., Davidson C.M. and Thomas R.P., Single and sequential extraction schemes for trace metal speciation in soil and sediment. In quality Assurance for environmental analysis (BCR), Quevauviller Ph., Maier E.A. and Griepink B. eds., Elsevier, 1995, pp 505-523.

Usero J., Gamero M., Morillo J. and Garcia I., Comparative study of three sequential extraction procedure for metals in marine sediments, Environment International, 24 (4) (1998) 487-496.

\section{$\underline{\text { Figures }}$}

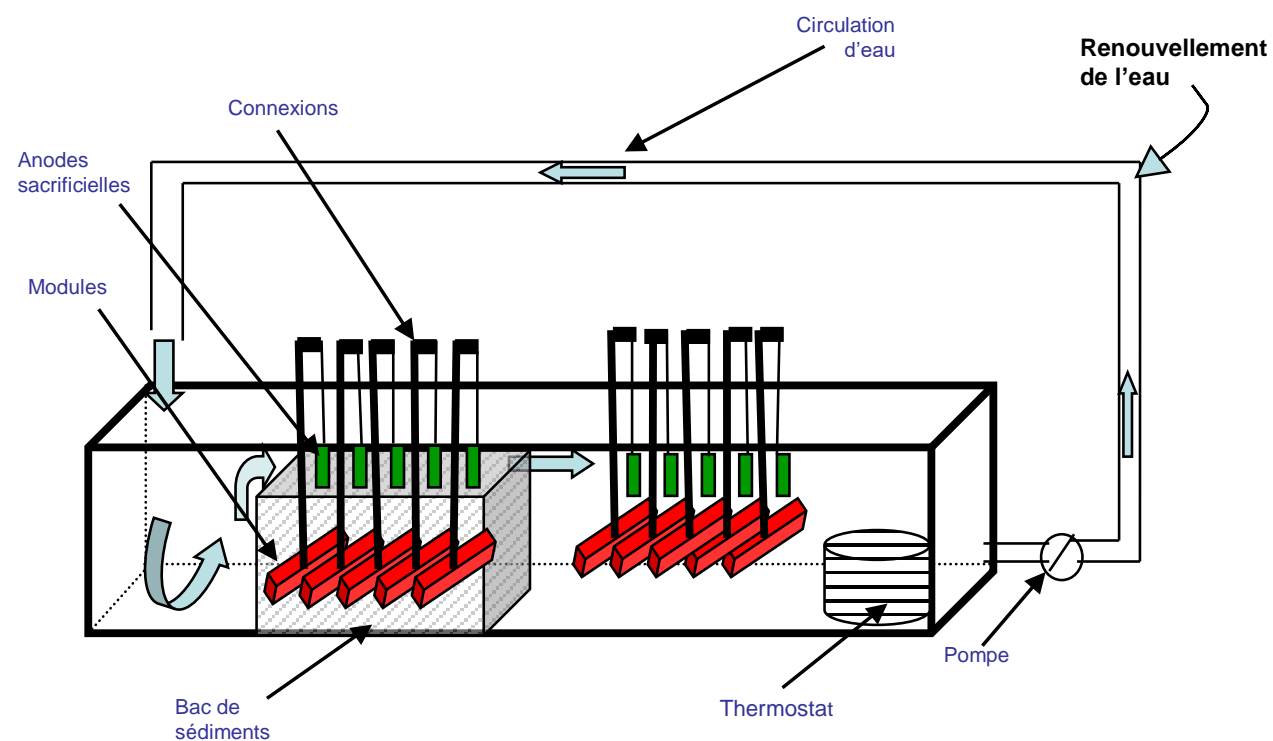

Figure 1. Schéma du bassin expérimental.

Scheme of the experimental basin

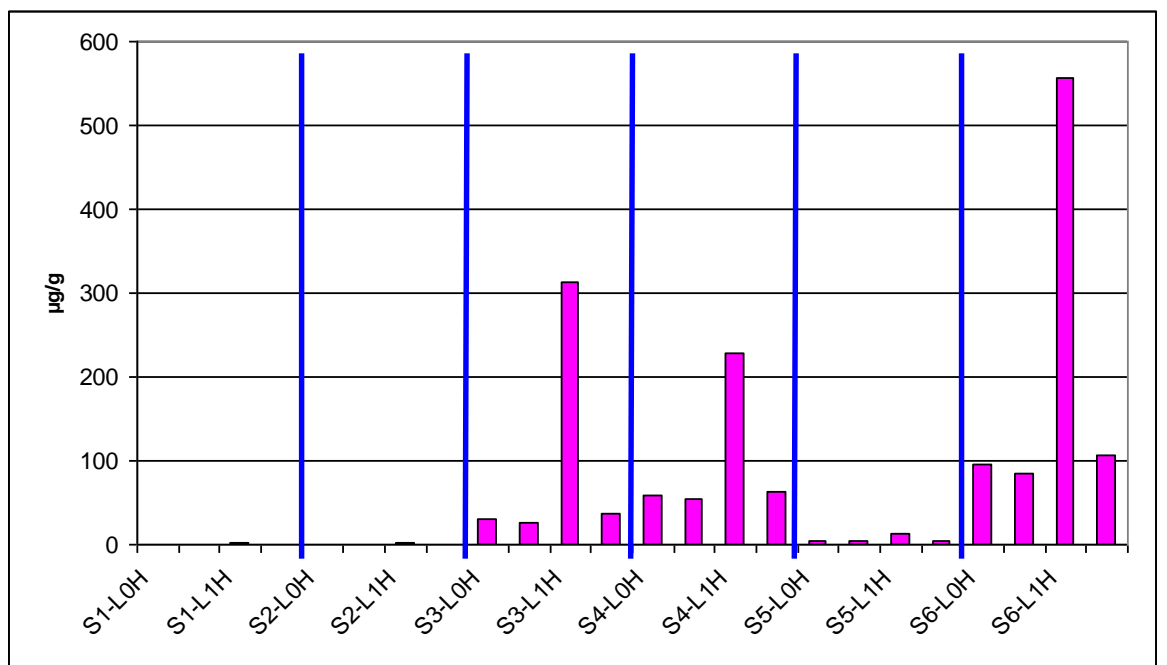

Figure 2: Quantité (en $\mu$ g) de zinc libéré, par gramme de sédiment sec, par les différentes fractions minéralogiques pour les 4 échantillons étudiés.

Quantity of zinc ( $\mu \mathrm{g}$ per $\mathrm{g}$ of dry sediment) leachable from the different mineralogical fractions 
Tableaux

Tableau 1 : Concentration en $\mathrm{mg} \cdot \mathrm{L}^{-1}$ dans la phase aqueuse au début (aq0) et à la fin de l'expérience (aq1)

Chemical composition of sea water in $\mathrm{mg} \cdot \mathrm{L}^{-1}$ (experiment beginning : aq 0 and experiment end: aq1)

\begin{tabular}{|c|c|c|c|c|c|c|c|c|c|c|c|c|c|c|}
\hline & $\mathrm{Al}$ & $\mathrm{Ca}$ & $\mathrm{Cd}$ & Co & $\mathrm{Cr}$ & $\mathrm{Cu}$ & $\mathrm{Fe}$ & K & $\mathrm{Mg}$ & $\mathrm{Mn}$ & $\mathrm{Ni}$ & & $\mathrm{Sr}$ & $\mathrm{Zn}$ \\
\hline $\mathrm{ac}$ & 0,01 & 349 & 0,000 & 0,003 & 0,000 & 0,01 & 0,000 & 11 & 940 & 0,001 & 0,004 & 0,004 & 2,7 & 0,6 \\
\hline $\mathrm{aq} 1$ & 0,01 & 364 & 0,000 & 0,001 & 0,000 & 0,01 & 0,000 & 119 & 944 & 0,001 & 0,000 & 0,008 & 2,8 & 0,5 \\
\hline
\end{tabular}

Tableau 2. Pourcentage d'oxydes présents dans les sédiments.

Oxides percentage in sediments

\begin{tabular}{|llllllll|}
\hline & $\mathrm{Al}_{2} \mathrm{O}_{3}$ & $\mathrm{CaO}$ & $\mathrm{Fe}_{2} \mathrm{O}_{3}$ & $\mathrm{~K}_{2} \mathrm{O}_{5}$ & $\mathrm{MgO}$ & $\mathrm{MnO}$ & $\mathrm{P}_{2} \mathrm{O}_{5}$ \\
$\mathrm{~L} 0 \mathrm{H}$ & 4 & 18 & 6 & 1,7 & 1,2 & 0,04 & 0,5 \\
$\mathrm{~L} 0 \mathrm{M}$ & 4 & 19 & 6 & 1,5 & 1,0 & 0,04 & 0,5 \\
$\mathrm{~L} 1 \mathrm{H}$ & 5 & 14 & 6 & 1,7 & 1,2 & 0,04 & 0,5 \\
$\mathrm{~L} 1 \mathrm{M}$ & 5 & 19 & 7 & 1,7 & 1,2 & 0,04 & 0,5 \\
Stevenson & 9 & 16 & 4 & 1,9 & 1,8 & 0,04 & 0,1 \\
$(2001)$ & & & & & & & \\
\hline
\end{tabular}

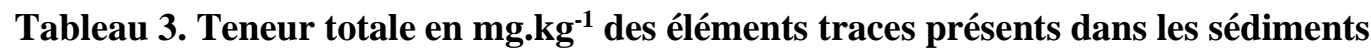
Chemical composition in trace elements in mg. $\mathrm{kg}^{-1}$ in sediments.

\begin{tabular}{|l|l|l|l|l|l|l|l|l|l|}
\hline & Cd & Co & Cr & Cu & Ni & Pb & Sc & Sr & Zn \\
\hline L0H & 1 & 7 & 36 & 26 & $<$ & 37 & 2 & 426 & 138 \\
\hline L0M & 1 & 7 & 35 & 24 & $<$ & 37 & 2 & 437 & 126 \\
\hline L1H & 1 & 7 & 39 & 26 & $<$ & 38 & 3 & 436 & 757 \\
\hline L1M & 1 & 7 & 40 & 27 & $<$ & 40 & 3 & 458 & 135 \\
\hline $\begin{array}{l}\text { Arrêté du } \\
14 / 06 / 2000\end{array}$ & 1 & $/$ & 90 & 45 & 37 & 100 & $/$ & $/$ & 276 \\
\hline $\begin{array}{l}\text { Stevenson } \\
(2001)\end{array}$ & $/$ & 7 & 55 & 15 & 32 & 11 & $/$ & 530 & 57 \\
\hline
\end{tabular}


Tableau 4 : Pourcentage de chaque élément solubilisé au cours de l'extraction séquentielle par rapport à sa quantité initialement présente.

Percentage of leached element in the different fractions (S1 to S6)

\begin{tabular}{|llllllllllllll|}
\hline & $\mathrm{Ca}$ & $\mathrm{Cd}$ & $\mathrm{Cr}$ & $\mathrm{Cu}$ & $\mathrm{Fe}$ & $\mathrm{K}$ & $\mathrm{Mn}$ & $\mathrm{P}$ & $\mathrm{Pb}$ & $\mathrm{S}$ & $\mathrm{Sc}$ & $\mathrm{Sr}$ & $\mathrm{Zn}$ \\
\hline L0M_S1 & 1 & 0 & 0 & 1 & 0 & 25 & 0 & 1 & 0 & 46 & 0 & 2 & 0 \\
L0H_S1 & 1 & 0 & 0 & 1 & 0 & 23 & 0 & 1 & 0 & 48 & 0 & 3 & 0 \\
L1H_S1 & 2 & 0 & 0 & 0 & 0 & 23 & 1 & 1 & 0 & 53 & 0 & 4 & 0 \\
L1M_S1 & 1 & 0 & 0 & 5 & 0 & 27 & 0 & 1 & 0 & 53 & 0 & 4 & 0 \\
\hline L0M_S2 & 2 & 0 & 0 & 2 & 0 & 19 & 3 & 2 & 0 & 10 & 0 & 6 & 0 \\
L0H_S2 & 2 & 0 & 0 & 1 & 0 & 19 & 3 & 2 & 0 & 11 & 0 & 6 & 0 \\
L1H_S2 & 2 & 0 & 0 & 1 & 0 & 16 & 2 & 2 & 0 & 10 & 0 & 6 & 0 \\
L1M_S2 & 2 & 0 & 0 & 2 & 0 & 17 & 2 & 2 & 0 & 12 & 0 & 6 & 0 \\
\hline L0M_S3 & 52 & 9 & 3 & 5 & 2 & 13 & 44 & 9 & 22 & 8 & 2 & 51 & 21 \\
L0H_S3 & 52 & 9 & 3 & 6 & 2 & 11 & 44 & 8 & 24 & 8 & 3 & 50 & 23 \\
L1H_S3 & 66 & 8 & 3 & 4 & 2 & 9 & 44 & 8 & 23 & 7 & 2 & 49 & 41 \\
L1M_S3 & 53 & 9 & 3 & 7 & 2 & 10 & 45 & 9 & 28 & 7 & 2 & 50 & 26 \\
\hline L0M_S4 & 3 & 43 & 41 & 36 & 46 & 9 & 19 & 51 & 7 & 8 & 44 & 3 & 43 \\
L0H_S4 & 5 & 41 & 41 & 40 & 44 & 8 & 19 & 59 & 7 & 7 & 43 & 5 & 42 \\
L1H_S4 & 6 & 42 & 38 & 42 & 42 & 7 & 18 & 55 & 6 & 8 & 36 & 4 & 30 \\
L1M_S4 & 4 & 48 & 44 & 41 & 47 & 8 & 21 & 56 & 6 & 6 & 40 & 4 & 44 \\
\hline L0M_S5 & 0 & 9 & 23 & 10 & 8 & 2 & 3 & 3 & 7 & 11 & 9 & 1 & 4 \\
L0H_S5 & 0 & 6 & 21 & 12 & 6 & 3 & 2 & 2 & 8 & 10 & 9 & 1 & 3 \\
L1H_S5 & 0 & 8 & 19 & 10 & 8 & 3 & 3 & 2 & 8 & 9 & 8 & 0 & 2 \\
L1M_S5 & 0 & 9 & 19 & 14 & 8 & 3 & 3 & 2 & 7 & 8 & 8 & 0 & 4 \\
\hline L0M_S6 & 58 & 61 & 67 & 54 & 56 & 68 & 69 & 66 & 36 & 83 & 56 & 63 & 68 \\
L0H_S6 & 60 & 56 & 64 & 58 & 52 & 64 & 69 & 71 & 39 & 85 & 54 & 64 & 69 \\
L1H_S6 & 75 & 58 & 60 & 57 & 51 & 58 & 68 & 68 & 37 & 86 & 46 & 63 & 74 \\
L1M_S6 & 60 & 66 & 65 & 69 & 57 & 65 & 71 & 70 & 41 & 86 & 51 & 64 & 74 \\
\hline moyenne S6 & 63 & 60 & 64 & 60 & 54 & 64 & 69 & 69 & 38 & 85 & 52 & 63 & 71 \\
écart type S6 & 8 & 4 & 3 & 7 & 3 & 4 & 1 & 2 & 2 & 2 & 4 & 1 & 3 \\
\hline
\end{tabular}

